\title{
REFLECTORS AS COMPOSITIONS OF EPI-REFLECTORS
}

\author{
BY \\ S. BARON $\left({ }^{1}\right)$
}

1. Introduction. This paper utilizes as a principal tool the factorization of every reflector as the composition of two epi-reflectors in categories satisfying certain general conditions. A prior result gives necessary and sufficient conditions for a subcategory to be epi-reflective. These lead to necessary and sufficient conditions for a subcategory to be cowell powered reflective. If the class of categories is further restricted, without however excluding any of those that arise naturally from classical mathematics, it is possible to exhibit factorizations of every reflector into two epi-reflectors through a greatest and a smallest intermediate category. Finally, some results are obtained concerning the generation and intersection of epi-reflective and reflective subcategories.

Definition. A subcategory $\mathscr{A}$ of a category $\mathscr{B}$ is said to be reflective if to every $\mathscr{B}$-object $B$, there corresponds an $\mathscr{A}$-object $R(B)$ known as the reflection of $B$ in $\mathscr{A}$ and a reflection map $r(B): B \rightarrow R(B)$, such that for any map $f$ from $B$ to an $\mathscr{A}$-object $A$, there is a unique map $R(f): R(B) \rightarrow A$ satisfying the equation $f=R(f) \circ r(B)$. We may extend $R$ to a functor from $\mathscr{B}$ to $\mathscr{A}$ by letting $R(g)=R\left(r\left(B_{2}\right) \circ g\right)$ where $g: B_{1} \rightarrow B_{2}$ is any $\mathscr{B}$-map. $R$ is then called the reflector from $\mathscr{B}$ to $\mathscr{A}$. Note that for a given reflective subcategory, the reflector and the reflection maps are unique up to isomorphism. If each reflection map is epi(mono), $\mathscr{A}$ is said to be an epi(mono)-reflective subcategory of $\mathscr{B}$ and $R$ is said to be an epi(mono)-reflector.

For the basic definitions of category theory, see Freyd [1]. To simplify the statement of theorems and definitions, all subcategories will be assumed to be full and replete. Terms will be prefixed by the name of the relevant category whenever necessary for emphasis or precision.

This paper was primarily inspired by a very interesting paper by Kennison [2], in which he defines and characterizes three types of reflective subcategory of $\mathscr{T}$, the category of topological spaces. Previously, Freyd [1, p. 87] had given sufficient conditions for a subcategory to be reflective, which were subsequently generalized by Isbell [4, p. 1276] in a bicategorical setting. Kennison modified Freyd's results to obtain necessary and sufficient conditions for epi-reflective subcategories of $\mathscr{T}$ and $\mathscr{T}_{2} \cdot\left(\mathscr{T}_{i}\right.$ is the category of $T_{i}$ spaces.) My first result is thus a generalization of

Presented to the Society, August 31, 1968; received by the editors October 20, 1967.

$\left.{ }^{1}\right)$ The author wishes to express his sincere thanks to his research director, Professor B. Rattray for his most valuable guidance, encouragement and constructive criticism throughout the preparing of the author's dissertation on which this paper is based. 
Kennison's results to a large class of categories. The method of factorization of reflectors was obtained independently by Kennison [3] for a different class of categories. He also has a more general bicategorical version of my Theorem 4 . The generation and intersection problems were posed by Isbell [5, p. 33, Problem 8] with regard to reflective subcategories of the category of uniform spaces.

The author wishes to thank the referee for strengthening the statements of Theorems 7 and 8.

2. Statement of results. Let $\mathscr{A}$ be a subcategory of $\mathscr{B}$.

Definition $1 . \mathscr{B}$ is said to be $\mathscr{A}$-cowell powered if for each $\mathscr{B}$-object $B$, the class of those $\mathscr{A}$-objects that are quotient objects of $B$ has a representative set.

Definition 2. $\mathscr{A}$ is said to be a quasi-epi-reflective (q e r) subcategory of $\mathscr{B}$ if for any map $f \in \mathscr{B}(B, A)$ where $A$ is an $\mathscr{A}$-object, there is an $\mathscr{A}$-object $A^{\prime}$ and maps $f_{1} \in\left(B, A^{\prime}\right), f_{2} \in\left(A^{\prime}, A\right)$ such that $f_{1}$ is epi and $f=f_{2} \circ f_{1}$.

Definition 3. A factorable category is one in which every map may be factored as an epi followed by a mono.

The first three theorems treat the problem of characterization of a reflective subcategory; the final result is Theorem 3, which characterizes a cowell powered, reflective subcategory of a factorable, cowell powered category with products.

THEOREM 1. Let $\mathscr{B}$ be an $\mathscr{A}$-cowell powered category with products. $\mathscr{A}$ is a $\mathscr{B}$-epireflective subcategory of $\mathscr{B}$ iff (1) $\mathscr{A}$ contains all $\mathscr{B}$-products of $\mathscr{A}$-objects and (2) $\mathscr{A}$ is a q e r subcategory of $\mathscr{B}$.

In Theorems 2 and $3, \mathscr{C}$ will be a factorable, cowell powered category with products.

THEOREM 2. Let $\mathscr{A}$ be a reflective subcategory of $\mathscr{C}$ and let $\mathscr{B}$ be the subcategory of $\mathscr{C}$ whose objects are the $\mathscr{C}$-subobjects of $\mathscr{A}$-objects. $\mathscr{A}$ is a $\mathscr{B}$-epi-reflective subcategory of $\mathscr{B}$ and $\mathscr{B}$ is a $\mathscr{C}$-epi-reflective subcategory of $\mathscr{C}$.

THEOREM 3. Let $\mathscr{A}$ be a subcategory of $\mathscr{C}$ and let $\mathscr{B}$ be the subcategory of $\mathscr{C}$ whose objects are the $\mathscr{C}$-subobjects of $\mathscr{A}$-objects. $\mathscr{A}$ is a cowell powered, reflective subcategory of $\mathscr{C}$ iff (1) $\mathscr{B}$ is $\mathscr{A}$-cowell powered, (2) $\mathscr{A}$ contains each $\mathscr{C}$-product of $\mathscr{A}$-objects and (3) $\mathscr{A}$ is a $q$ e r subcategory of $\mathscr{B}$.

Definition 4. A mono $f: A \rightarrow B$ is said to be extremal if in any factorization $f=g \circ e$ where $e$ is epi, $e$ must be iso. $(A, f)$ is said to be an extremal subobject of $B$.

Theorem 4 is a more convenient characterization of epi-reflective subcategories and utilizes the theory of extremal subobjects that is developed in $\S 6$.

THEOREM 4. Let $\mathscr{B}$ be a left complete, well and $\mathscr{A}$-cowell powered category. $\mathscr{A}$ is an epi-reflective subcategory of $\mathscr{B}$ iff $\mathscr{A}$ contains all $\mathscr{B}$-products and $\mathscr{B}$-extremal subobjects of its objects. 
Definition 5. Let $R$ be a reflector from $\mathscr{C}$ to $\mathscr{A}$. If $R=S T$ where $T: \mathscr{C} \rightarrow \mathscr{B}$ is a $\mathscr{C}$-epi-reflector and $S: \mathscr{B} \rightarrow \mathscr{A}$ is a $\mathscr{B}$-epi-reflector, then $\mathscr{B}$ is said to be an intermediate category of the pair $(\mathscr{A}, \mathscr{C})$.

Thus Theorem 2 asserts that the subcategory whose objects are the $\mathscr{C}$-subobjects of $\mathscr{A}$-objects is an intermediate category of $(\mathscr{A}, \mathscr{C})$. Theorems 5 and 6 show that under certain conditions there are greatest and smallest intermediate categories.

THEOREM 5. Let $\mathscr{A}$ be a reflective subcategory of a well powered category $\mathscr{C}$ with intersections and difference kernels. The subcategory $\mathscr{B}^{\prime}$ whose objects are the $\mathscr{C}$ extremal subobjects of the $\mathscr{A}$-objects is the smallest intermediate subcategory of $(\mathscr{A}, \mathscr{C})$.

Theorem 6. Let $\mathscr{A}, \mathscr{B}^{\prime}$ and $\mathscr{C}$ be as in Theorem 5 and let $\mathscr{C}$ be, in addition, cowell powered and left complete. Define $\mathscr{B}^{\prime \prime}$, a subcategory of $\mathscr{C}$, as follows: $B^{\prime \prime}$ is a $\mathscr{B} "$ "object iff for each $\mathscr{B}^{\prime}$-object $B^{\prime}$ and pair of maps $f_{1}, f_{2}: R\left(B^{\prime}\right) \rightarrow B^{\prime \prime}, f_{1} \circ r\left(B^{\prime}\right)=f_{2} \circ r\left(B^{\prime}\right)$ $\Rightarrow f_{1}=f_{2}$. (Here, $R$ is the reflector from $\mathscr{B}^{\prime}$ to $\mathscr{A}$ and $r\left(B^{\prime}\right): B^{\prime} \rightarrow R\left(B^{\prime}\right)$ is the corresponding reflection map.) $\mathscr{B}^{\prime \prime}$ is then the largest intermediate category of $(\mathscr{A}, \mathscr{C})$.

The final two theorems concern the generation and intersection of reflective subcategories. $\mathscr{C}$ will be assumed to be a left complete, well and cowell powered category.

THEOREM 7. Let $\mathscr{S}$ be a subcategory of $\mathscr{C}$. Let $\mathscr{B}$ be the subcategory of $\mathscr{C}$ whose objects are the $\mathscr{C}$-extremal subobjects of products of $\mathscr{S}$-objects and $\mathscr{A}$ be the subcategory of $\mathscr{B}$ whose objects are the $\mathscr{B}$-extremal subobjects of products of $\mathscr{S}$-objects.

(1) $\mathscr{B}$ is the smallest epi-reflective subcategory of $\mathscr{C}$ that contains $\mathscr{S}$.

(2) If $\mathscr{D}$ is any reflective subcategory of $\mathscr{C}$ and if $\mathscr{D}$ contains $\mathscr{S}$, then $\mathscr{D}$ also contains $\mathscr{A}$. Thus a necessary condition for $\mathscr{S}$ to be reflective is that $\mathscr{S}=\mathscr{A}$.

(3) If $\mathscr{B}$ is cowell powered, then $\mathscr{A}$ is the smallest reflective subcategory of $\mathscr{C}$ that contains $\mathscr{S}$.

THEOREM 8. Let $\left\{\mathscr{A}_{i}\right\}$ be a class of reflective subcategories of $\mathscr{C}$.

(1) If each $\mathscr{A}_{i}$ is epi-reflective, then $\bigcap \mathscr{A}_{i}$ is epi-reflective.

(2) If $\mathscr{B}$ is cowell powered and an intermediate category of each $\left(\mathscr{A}_{i}, \mathscr{C}\right)$, then $\cap \mathscr{A}_{i}$ is reflective.

(3) If $\mathscr{B}_{i}$ is an intermediate category of $\left(\mathscr{A}_{i}, \mathscr{C}\right)$ for each $i$ and $\cap \mathscr{B}_{i}$ is cowell powered, then $\cap \mathscr{A}_{i}$ is reflective and $\cap \mathscr{B}_{i}$ is an intermediate category of $\left(\cap \mathscr{A}_{i}, \mathscr{C}\right)$.

3. Proof of Theorem 1. $\Rightarrow$ (1) It can be shown that when the $\mathscr{B}$-left root of an $\mathscr{A}$-diagram is reflected in $\mathscr{A}$, the reflection map is an isomorphism. This method does not depend upon the conditions on $\mathscr{A}$ or $\mathscr{B}$.

(2) If $f$ maps a $\mathscr{B}$-object $B$ to an $\mathscr{A}$-object $A$, then $f=R(f) \circ r(B)$ where $r(B)$ is an epi that maps $B$ to an $\mathscr{A}$-object $R(B)$.

$\Leftrightarrow$ Let $B$ be a $\mathscr{B}$-object. Since $\mathscr{B}$ is $\mathscr{A}$-cowell powered, the class of quotient objects of $B$ that are also $\mathscr{A}$-objects has a representative set, $\left\{B_{\lambda}\right\}$. Thus we may form 
$\prod B_{\lambda}$ which is an $\mathscr{A}$-object and we have an evaluation map $e: B \rightarrow \prod B_{\lambda}$. Using the fact that $\mathscr{A}$ is q e r, we factor $e$ as $g \circ r(B)$ where $r(B): B \rightarrow R(B)$ is epi and $R(B)$ is an $\mathscr{A}$-object.

We now show that $R(B)$ is the reflection of $B$ and $r(B)$ is the reflection map. Let $f: B \rightarrow A$ where $A$ is an $\mathscr{A}$-object. Since $\mathscr{A}$ is q e r, $f$ may be factored through a $B_{\lambda}$, hence through $\Pi B_{\lambda}$ via the evaluation map and thus through $R(B)$ via $r(B)$. $R(f)$ is the composition of the other three maps on the perimeter of the square in the diagram. It is clear that $f=R(f) \circ r(B)$ and $R(f)$ is unique because $r(B)$ is epi. Thus every $\mathscr{B}$-object has a reflection in $\mathscr{A}$.

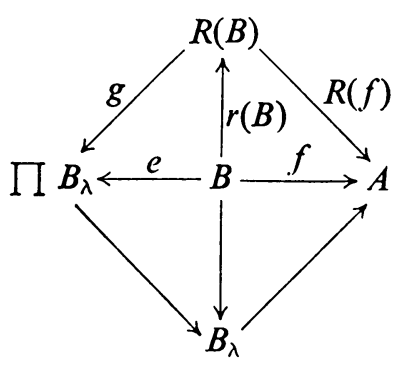

4. Proof of Theorem 2. Since $\mathscr{A}$ is reflective, it contains all $\mathscr{C}$-products of its objects; since the product of subobjects of a set of objects is a subobject of the product of the objects, $\mathscr{B}$ contains all $\mathscr{C}$-products of its objects.

Since $\mathscr{C}$ is factorable, any $\mathscr{C}$-map may be factored as an epi followed by a mono. In particular, any $\mathscr{C}$-map whose range is a $\mathscr{B}$-object may be factored via an epi through a subobject of the range. Since subobjects of $\mathscr{B}$-objects are $\mathscr{B}$-objects, $\mathscr{B}$ is a $\mathrm{q} \mathrm{e} \mathrm{r}$ and hence an epi-reflective subcategory of $\mathscr{C}$.

$\mathscr{A}$ is clearly a reflective subcategory of $\mathscr{B}$. We must now show that $r(B)$ is $\mathscr{B}$-epi for each $\mathscr{B}$-object $B$.

Suppose $f, g: R(B) \rightarrow B^{\prime}$ are two $\mathscr{B}$-maps such that $f \circ r(B)=g \circ r(B)$. Let $m$ be the subobject map from $B^{\prime}$ to some $\mathscr{A}$-object, $A$. Then $m \circ f \circ r(B)=m \circ g \circ r(B): B \rightarrow A$. Since this map must have a unique reflection, it follows that $m \circ f=m \circ g$. $f=g$ because $m$ is mono and $\mathscr{A}$ is a $\mathscr{B}$-epi reflective subcategory of $\mathscr{B}$.

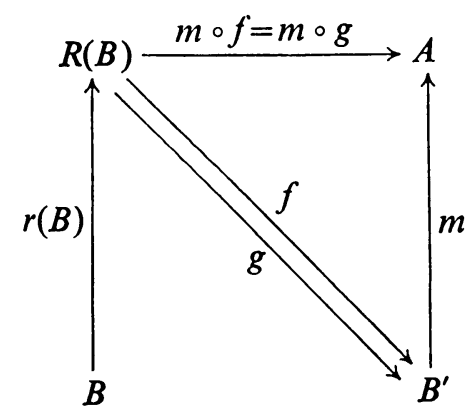


5. Proof of Theorem 3. $\Rightarrow(2)$ is clear as in $\S 3$. By Theorems 2 and 1 , we have (3). It can be shown that if $(A, f)$ is a $\mathscr{B}$-quotient object of $B$, then $(A, R(f))$ is an $\mathscr{A}$-quotient object of $R(B)$. Since $\mathscr{A}$ is cowell powered, (1) follows.

$\Leftrightarrow$ Since $\mathscr{C}$ has products, it follows from (2) that $\mathscr{A}$ contains each $\mathscr{B}$-product of $\mathscr{A}$-objects. Using (3), it follows from Theorem 1 that $\mathscr{A}$ is a reflective subcategory of $\mathscr{B}$.

$\mathscr{B}$ is a q e r subcategory of $\mathscr{C}$ as in $\$ 4$. Using (2), we may show, as in $\S 4$, that $\mathscr{B}$ contains each $\mathscr{C}$-product of $\mathscr{B}$-objects; by Theorem $1, \mathscr{B}$ is a reflective subcategory of $\mathscr{C}$. Thus $\mathscr{A}$ is a reflective subcategory of $\mathscr{C}$.

We may show that any $\mathscr{A}$-epi is a $\mathscr{B}$-epi; it then follows from (1) that $\mathscr{A}$ is cowell powered.

6. Extremal subobjects. Before proceeding to the proof of Theorem 4, it will be necessary to develop part of the theory of extremal subobjects. These results are contained in a paper by Isbell [6], but will be obtained somewhat differently, here.

\subsection{A regular subobject is extremal.}

Proof. By a regular subobject, we mean a subobject that is the difference kernel of two maps. If $(A, f)$ is the difference kernel of $\left(g_{1}, g_{2}\right)$ and $f=m \circ e$ where $e$ is epi, then $g_{1} \circ m=g_{2} \circ m$. Thus, there is an $h$ such that $m=f \circ h$. It can be shown that $h$ is the inverse of $e$ and thus $(A, f)$ is extremal.

6.2. In a well powered category with intersections and difference kernels, if $f \circ e=m \circ g$ where $e: A \rightarrow B$ is epi and $m: C \rightarrow D$ is extremal mono, then there is a map $h: B \rightarrow C$ such that $h \circ e=g$ and $m \circ h=f$.

Proof. Let $(I, x)$ be the intersection of those subobjects of $D$ through which both $m$ and $f$ factor. Let $m=x \circ y$ and $f=x \circ z . x \circ z \circ e=f \circ e=m \circ g=x \circ y \circ g$. Since

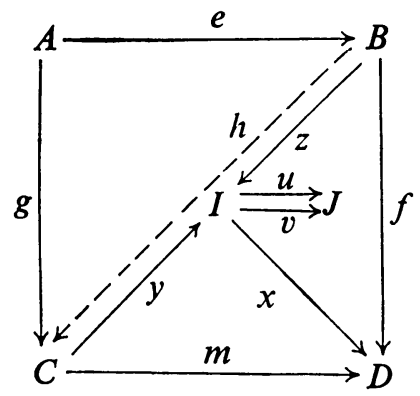

$x$ is mono, $z \circ e=y \circ g$. Suppose $u, v: I \rightarrow J$ such that $u \circ y=v \circ y$. Then, $u \circ z \circ e$ $=u \circ y \circ g=v \circ y \circ g=v \circ z \circ e$; since $e$ is epi, $u \circ z=v \circ z$. Thus, $y$ and $z$ both factor through the difference kernel of $(u, v)$. One may show that this implies that this difference kernel is isomorphic with $I$; whence, $y$ is epi. Since $m$ is extremal mono, $y$ must be iso. Let $h=y^{-1} \circ z$. Now $f=x \circ z=x \circ y \circ y^{-1} \circ z=m \circ h$. Also $m \circ g$ $=f \circ e=m \circ h \circ e$. Thus $g=h \circ e$.

We now have the major lemma of this section. 
6.3. Let $\mathscr{D}$ be a small category and $\mathscr{A}$ be a well powered category with intersections and difference kernels. If two functors $F_{1}, F_{2}: \mathscr{D} \rightarrow \mathscr{A}$ have left roots and a natural transformation $\mu: F_{1} \rightarrow F_{2}$ such that for each $\mathscr{D}$-object $D, \mu(D)$ is an extremal mono, then the induced morphism $f$, mapping the left root of $F_{1}$ to the left root of $F_{2}$, is an extremal mono.

Proof. We show first that $f$ is mono. Let $\left(L_{i}, \phi_{i}\right)$ be the left root of $F_{i}$. Suppose $f \circ x=f \circ y$, where $x, y: A \rightarrow L_{i}$. Then

$$
\mu\left(D_{\lambda}\right) \circ \phi_{1}\left(D_{\lambda}\right) \circ x=\phi_{2}\left(D_{\lambda}\right) \circ f \circ x=\phi_{2}\left(D_{\lambda}\right) \circ f \circ y=\mu\left(D_{\lambda}\right) \circ \phi_{1}\left(D_{\lambda}\right) \circ y .
$$

Since $\mu\left(D_{\lambda}\right)$ is mono, it follows that $\phi_{1}\left(D_{\lambda}\right) \circ x=\phi_{1}\left(D_{\lambda}\right) \circ y$. It can be shown that these maps are consistent with $F_{1}$. By the definition of "left root", there must be a unique map from $A$ to $L_{1}$ which when composed with each $\phi_{1}\left(D_{\lambda}\right)$ yields the corresponding $\phi_{1}\left(D_{\lambda}\right) \circ x$. Thus $x=y$ and $f$ is mono.

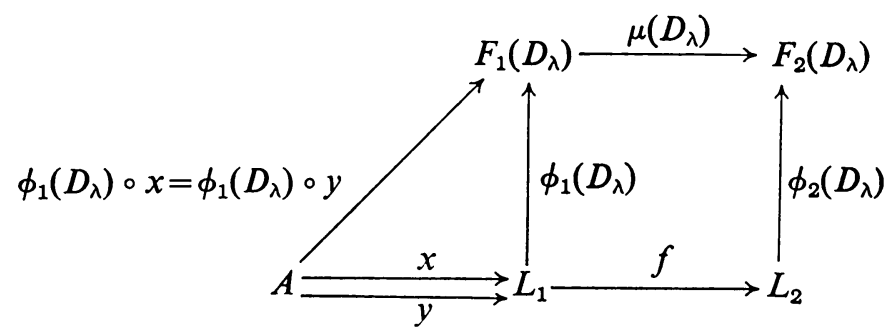

We now show that $f$ is extremal. Suppose $f=x \circ e$, where $e: L_{1} \rightarrow C$ is epi. Since each $\mu\left(D_{\lambda}\right)$ is extremal mono, we may apply 6.2 to obtain maps $h_{\lambda}: C \rightarrow F_{1}\left(D_{\lambda}\right)$. These induce a map $h: C \rightarrow L_{1}$ which can be shown to be the inverse of $e$.

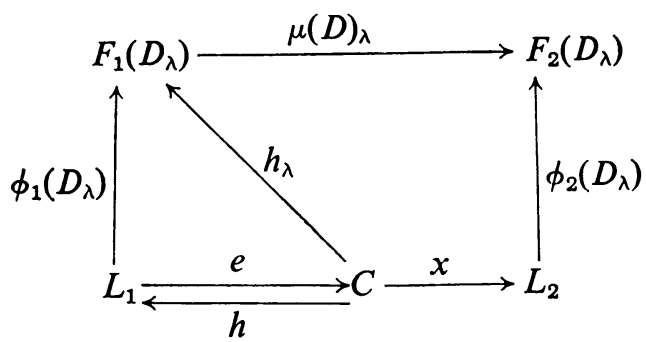

The preceding result is equivalent to the statement that the extremal monos are closed under the formation of left roots in Morph $(\mathscr{A})$.

COROLlaRY 1. In a category satisfying the above conditions, the product of extremal subobjects of a set of objects is an extremal subobject of the product of the set, each containing object being taken as often as it occurs.

COROLlary 2. In a category satisfying the above conditions, the intersection of a family of extremal subobjects of a given object is again an extremal subobject. 
Proof. The subobject maps define a natural transformation from the diagram determined by the family of extremal subobjects, to the constant diagram consisting of the object. Since the left root of the first diagram is the intersection of the extremal subobjects and the left root of the constant diagram is itself, it follows from 6.3 that there must be an extremal mono from the intersection to the containing object.

6.4. In a well powered category with intersections and difference kernels, the composition of two extremal monos is an extremal mono.

Proof. Suppose $g \circ f=h \circ e$ where $g$ and $f$ are extremal monos and $e$ is epi. By 6.2, $f$ factors through $e$ and therefore, $e$ is iso.

6.5. In a well powered category with intersections and difference kernels, any map may be factored as an epi followed by an extremal mono.

Proof. Consider a map, $f: A \rightarrow B$; let $(C, m)$ be the intersection of those extremal subobjects of $B$ through which $f$ factors. Suppose $f=m \circ g$. By 6.3 Corollary $2, m$ is an extremal mono. Using 6.1 and the method of $6.2, g$ can be shown to be epi.

7. Proof of Theorem 4. In view of Theorem 1, we need only show that, under the conditions, $\mathscr{A}$ contains all $\mathscr{B}$-extremal subobjects of its objects iff $\mathscr{A}$ is a q e r subcategory.

$\Rightarrow$ Let $f: B \rightarrow A$ where $A$ is an $\mathscr{A}$-object. By 6.5, $f=m \circ e$ where $e$ is epi and $m$ is extremal mono. Using our hypothesis, we see that the range of $e$ is an $\mathscr{A}$-object and that $\mathscr{A}$ is a q e r subcategory.

$\Leftrightarrow$ Let $(B, m)$ be a $\mathscr{B}$-extremal subobject of an $\mathscr{A}$-object $A$. Since $\mathscr{A}$ is q e r, we have $m=f \circ g$ where $g$ is an epi whose range is an $\mathscr{A}$-object. Since $m$ is extremal, it follows that $g$ is iso and $B$ is an $\mathscr{A}$-object.

8. Proof of Theorem 5. Let $C$ be a $\mathscr{C}$-object with reflection map $r(C): C \rightarrow R(C)$, where $R$ is the reflector from $\mathscr{C}$ to $\mathscr{A}$. By 6.5 , we may factor $r(C)$ as $m \circ r^{\prime}(C)$ where $r^{\prime}(C): C \rightarrow R^{\prime}(C)$ is epi and $m: R^{\prime}(C) \rightarrow R(C)$ is mono. Using reflection properties, it may be shown that $R(C)=R\left(R^{\prime}(C)\right)$ and $m=r\left(R^{\prime}(C)\right)$. As in $\S 4, \mathscr{A}$ may be shown to be a $\mathscr{B}^{\prime}$-epi-reflective subcategory of $\mathscr{B}^{\prime}$.

It remains to show $R^{\prime}: \mathscr{C} \rightarrow \mathscr{B}^{\prime}$ is a reflector with reflection maps, $r^{\prime}(C)$. Let $f: C \rightarrow B^{\prime}$, where $B^{\prime}$ is a $\mathscr{B}^{\prime}$-object; say, $\left(B^{\prime}, m^{\prime}\right)$ is an extremal subobject of an $\mathscr{A}$-object, $A$. By the reflection property, there is an $R\left(m^{\prime} \circ f\right): R(C) \rightarrow A$ such that $R\left(m^{\prime} \circ f\right) \circ m \circ r^{\prime}(C)=m^{\prime} \circ f$. By 6.2, since $r^{\prime}(C)$ is epi and $m$ is extremal mono, there is an $R^{\prime}(f): R^{\prime}(C) \rightarrow B$ such that $R^{\prime}(f) \circ r^{\prime}(C)=f$. It is unique because $r^{\prime}(C)$ is epi. We have thus shown that $\mathscr{B}^{\prime}$ is an intermediate subcategory of $(\mathscr{A}, \mathscr{C})$.

Suppose $\mathscr{D}$ is an intermediate category of $(\mathscr{A}, \mathscr{C})$. In this case, $\mathscr{D}$ contains $\mathscr{A}$ and is a $\mathscr{C}$-epi-reflective subcategory of $\mathscr{C}$. By results in $\S \S 3$ and 7, $\mathscr{D}$ contains all of the $\mathscr{C}$-extremal subobjects of $\mathscr{C}$-products of its objects and, in particular, $\mathscr{D} \supseteq \mathscr{B}^{\prime}$. 


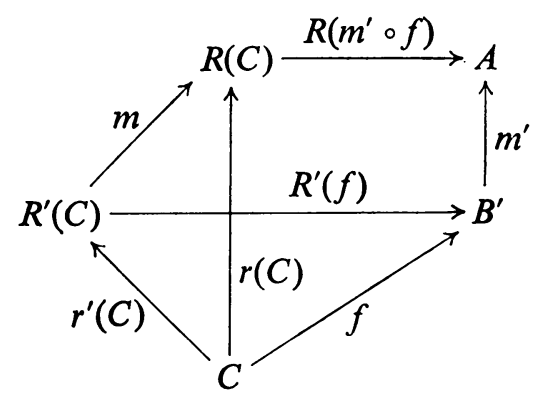

9. Proof of Theorem 6. It is not difficult to show that $\mathscr{B}^{\prime \prime}$ is closed under the formation of $\mathscr{C}$-products and $\mathscr{C}$-subobjects; $\mathscr{B}^{\prime \prime}$ is thus a $\mathscr{C}$-epi-reflective subcategory of $\mathscr{C}$.

Let $R^{\prime \prime}: \mathscr{B}^{\prime \prime} \rightarrow \mathscr{A}$ be the restriction of the reflector $R: \mathscr{C} \rightarrow \mathscr{A}$. It is clear that $R^{\prime \prime}$ is a reflector and we need only show that each reflection map $r^{\prime \prime}\left(B^{\prime \prime}\right)$ is $\mathscr{B}^{\prime \prime}$-epi. By 6.5, we have $r^{\prime \prime}\left(B^{\prime \prime}\right)=m \circ e$ where $e$ is $\mathscr{C}$-epi and $m$ is $\mathscr{C}$-extremal mono. $B^{\prime}$, the range of $e$ is a $\mathscr{B}^{\prime}$-object. One may show that $m=r^{\prime \prime}\left(B^{\prime}\right)=r\left(B^{\prime}\right)$ and $R^{\prime \prime}\left(B^{\prime \prime}\right)=R^{\prime \prime}\left(B^{\prime}\right)$ $=R\left(B^{\prime}\right)$, by using the reflection property.

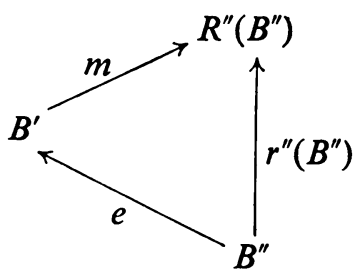

Thus, since $\mathscr{B}^{\prime \prime}$ was defined in such fashion that the reflection maps from $\mathscr{B}^{\prime}$-objects remain epi, $m$ is $\mathscr{B}^{\prime \prime}$-epi. Thus $r^{\prime \prime}\left(B^{\prime \prime}\right)$ is $\mathscr{B}^{\prime \prime}$-epi and $\mathscr{B}^{\prime \prime}$ is an intermediate category of $(\mathscr{A}, \mathscr{C})$.

Let $\mathscr{D}$ be an intermediate category of $(\mathscr{A}, \mathscr{C})$. By Theorem $5, \mathscr{D} \supseteq \mathscr{B}$. Since, in particular, the reflection maps of all of the $\mathscr{B}^{\prime}$-objects must be $\mathscr{D}$-epi, it follows that $\mathscr{D} \subseteq \mathscr{B} "$.

10. Proof of Theorem 7. (1) is a simple consequence of Theorem 4.

(2) Suppose $\mathscr{D}$ is a reflective subcategory containing $\mathscr{S}$. Let $\mathscr{E}$ be the smallest intermediate category of $(\mathscr{D}, \mathscr{C})$ as described in Theorem 5 . It follows from Theorem 5 , that $\mathscr{B} \subseteq \mathscr{E}$. Let $T$ be an $\mathscr{A}$-object: suppose $(T, f)$ is a $\mathscr{B}$-extremal subobject of $U$, a product of elements of $\mathscr{S}$. By 6.5, we may factor $f$ as $m \circ e$ where $e: T \rightarrow V$ is $\mathscr{E}$-epi and $m: V \rightarrow U$ is $\mathscr{E}$-extremal mono. ( $\mathscr{E}$ may be shown to be left complete and well powered.) Again by 6.5, factor $m$ as $m_{1} \circ e_{1}$ where $e_{1}: V \rightarrow W$ is $\mathscr{C}$-epi and $m_{1}: W \rightarrow U$ is $\mathscr{C}$-extremal mono. $W$ is a $\mathscr{B}$-object and thus an $\mathscr{E}$-object; therefore $e_{1}$ is iso and $V$ is a $\mathscr{B}$-object. We may now use the fact that $f$ is $\mathscr{B}$-extremal mono to obtain that $e$ is iso; it follows that $T$ is an $\mathscr{E}$-extremal subobject of $U$ and, by Theorem 4 , a $\mathscr{D}$-object. Thus $\mathscr{A} \subseteq \mathscr{D}$. 


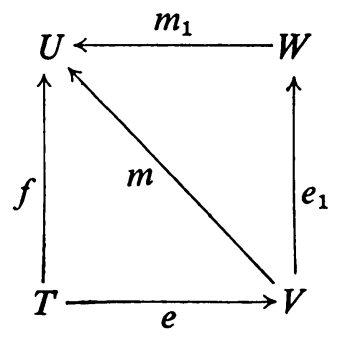

(3) $\mathscr{B}$ is an epi-reflective subcategory of $\mathscr{C}$. Since $\mathscr{C}$ is left complete and $\mathscr{B}$ is closed under the formation of $\mathscr{C}$-left roots of $\mathscr{B}$-diagrams, it follows that $\mathscr{B}$ is left complete. Finally, we show that all $\mathscr{B}$-monos are $\mathscr{C}$-monos, from which we establish that $\mathscr{B}$ is well-powered. Suppose $m: D \rightarrow B$ is a $\mathscr{B}$-mono and that $m \circ f=m \circ g$, where $f$ and $g$ map a $\mathscr{C}$-object $C$ to $D$. Reflect $C$ in $\mathscr{B}$ to obtain the diagram.

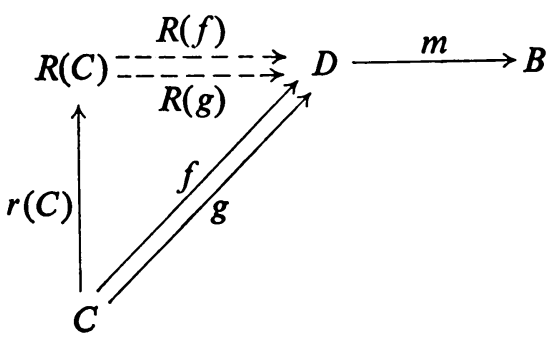

Now since $r(C)$ is epi, it follows that $m \circ R(f)=m \circ R(g)$. Since $m$ is a $\mathscr{B}$-mono, it follows that $R(f)=R(g)$ and $f=g$. Thus $\mathscr{B}$ and $\mathscr{A}$ satisfy the conditions of Theorem 4 and $\mathscr{A}$ is a reflective subcategory of $\mathscr{B}$ and thus of $\mathscr{C}$.

11. Proof of Theorem 8. (1) By Theorem 4, each $\mathscr{A}_{i}$ contains all $\mathscr{C}$-extremal subobjects and $\mathscr{C}$-products of its objects; hence so does $\bigcap \mathscr{A}_{i}$.

(2) As in $\$ 10, \mathscr{B}$ is left complete, and well and cowell powered. Thus by (1), $\cap \mathscr{A}_{i}$ is a $\mathscr{B}$-epi-reflective subcategory of $\mathscr{B}$ and a reflective subcategory of $\mathscr{C}$.

(3) By (1), $\bigcap \mathscr{B}_{i}$ is a $\mathscr{C}$-epi-reflective subcategory of $\mathscr{C}$ and as in $\S 10, \bigcap \mathscr{B}_{i}$ is left complete, and well and cowell powered. Each $\cap \mathscr{B}_{i}$-product is a $\mathscr{C}$-product; since each $\mathscr{A}_{i}$ contains all $\mathscr{C}$-products of its objects, $\cap \mathscr{A}_{i}$ contains all $\cap \mathscr{B}_{i}$-products of its objects. Let $(B, m)$ be an $\bigcap \mathscr{B}_{i}$-extremal subobject of an $\bigcap \mathscr{A}_{i}$-object, $A$. By factoring $m$ first in $\mathscr{B}_{i}$, then in $\mathscr{C}$, as an epi followed by an extremal mono, we may establish that $m$ is a $\mathscr{B}_{i}$-extremal mono. Hence, $B$ is an $\mathscr{A}_{i}$-object and, since this is true for all $i$, an $\cap \mathscr{A}_{i}$-object. Thus $\cap \mathscr{A}_{i}$ is an epi-reflective subcategory of $\cap \mathscr{B}_{i}$.

12. Applications to $\mathscr{T}$. The example which has motivated a good deal of the work on reflective subcategories is that of the category of compact-Hausdorff spaces, which is a reflective subcategory of $\mathscr{T}$. In this case, the smallest intermediate subcategory is the category of completely regular spaces; these are the $\mathscr{T}$-extremal subobjects (i.e. the subspaces) of the compact-Hausdorff spaces. The reflection 
of an object is then its Stone-Čech compactification. The largest intermediate category would be the category of those spaces which when they are the range of a map from a completely regular space do not permit extension of the map to its Stone-Čech compactification in more than one way. $\mathscr{T}_{2}$ is a proper subcategory of this category.

Kennison [2] mentions and characterizes two main types of reflective subcategory of $R$ : (1) those in which the reflection map is onto, (2) those which are contained in $\mathscr{T}_{2}$ and have dense reflection maps. The first of these is the class of epi-reflective subcategories and the second is the class of those reflective subcategories having $\mathscr{T}_{2}$ as an intermediate category. He asks if there might be other types. To produce another type, we need an intermediate category in which epi has a different meaning. This is provided by $\mathscr{T}_{0}$, in which $e: S \rightarrow T$ is epi iff for each $x \in T$, every neighborhood of $x$ intersects $x^{-} \cap e(S)$, [7]. An example of a reflective subcategory of this type is the category of $\mathscr{T}_{0}$-extremal subobjects of products of the connected doublet (the two point space with three open sets). This category is, incidentally, the smallest mono-reflective subcategory of $\mathscr{T}_{0}$. In the same way, the indiscrete spaces form the smallest mono-reflective subcategory of $\mathscr{T}$ and the compact-Hausdorff spaces, as Kennison has pointed out, form the smallest mono-reflective subcategory of the category of completely regular spaces. One may obtain a general result for any category which has a cogenerator which is an extremal subobject of any containing object.

Much work remains to be done on the problem of the classification of reflective subcategories of $\mathscr{T}$ and this will, perhaps, motivate more general results.

\section{BIBLIOGRAPHY}

1. P. Freyd, Abelian categories, Harper and Row, New York, 1964.

2. J. F. Kennison, Reflective functors in general topology and elsewhere, Trans. Amer. Math. Soc. 118 (1965), 303-315.

3. — Full reflective subcategories, Illinois J. Math 12 (1968), 353-365.

4. J. R. Isbell, Natural sums and abelianizing, Pacific J. Math. 14 (1964), 1265-1281.

5. — Uniform spaces, Math. Surveys, No. 12, Amer. Math. Soc., Providence, R. I., 1964.

6. - Subobjects, adequacy, completeness and categories of algebras, Rozprawy Mat. 36 (1964), 1-31.

7. S. Baron, Note on epi in $T_{0}$, Canad. Math. Bull. (to appear).

MCGILl UNIVERSITY, Montreal, Canada 